\title{
AN ALTERNATING PROCEDURE FOR OPERATORS ON UNIFORMLY CONVEX AND UNIFORMLY SMOOTH BANACH SPACES
}

\author{
ZONG-BEN XU AND G. F. ROACH
}

(Communicated by Palle E. T. Jorgensen)

\begin{abstract}
Let $X$ be a real uniformly convex and uniformly smooth Banach space. For any $1<p<\infty, J_{p}, J_{p}^{*}$ respectively denote the duality mapping with gauge function $\varphi(t)=t^{p-1}$ from $X$ onto $X^{*}$ and $X^{*}$ onto $X$. If $T: X \rightarrow X$ is a bounded linear operator, then $M(T): X \rightarrow X$ is the mapping defined by $M(T)=J_{q}^{*} T^{*} J_{p} T$, where $T^{*}: X^{*} \rightarrow X^{*}$ is the adjoint of $T$ and $q=(p-1)^{-1} p$. It is proved that if $T_{n}$ is a sequence of operators on $X$ such that $\left\|T_{n}\right\| \leq 1$ for all $n$, then $M\left(T_{n}, \ldots, T_{1}\right) x$ strongly converges in $X$ for any $x \in X$, with an estimate of the rate of convergence:

$$
\left\|M\left(T_{n} \cdots T_{1}\right) x-M(x)\right\| \leq \sigma(x)\|x\| \psi\left(1-\left(m(x) /\left\|T_{n} \cdots T_{1} x\right\|\right)^{p}\right),
$$

where $M(x)=\lim _{n \rightarrow \infty} M\left(T_{n} \cdots T_{1}\right) x, m(x)=\lim _{n \rightarrow \infty}\left\|T_{n} \cdots T_{1} x\right\|$, and $\sigma: X \rightarrow R^{+}, \psi: R^{+} \rightarrow R^{+}$are definite, strictly increasing positive functions. The result obtained generalizes and improves on the theorem offered recently by Akcoglu and Sucheston [1].
\end{abstract}

\section{INTRODUCTION}

Let $X$ be a real Banach space, with dual space $X^{*}$ and associated generalized duality pairing denoted by $\langle\cdot, \cdot\rangle$. Suppose that $X$ and $X^{*}$ are both uniformly convex (therefore, reflexive). We identify $X=X^{* *}$ in the usual way. For any $1<k<\infty$, let $J_{k}$ be defined by

$$
J_{k} x:=\left\{x^{*} \in X^{*}:\left\langle x^{*}, x\right\rangle=\left\|x^{*}\right\|\|x\|,\left\|x^{*}\right\|=\|x\|^{k-1}\right\} \quad \forall x \in X .
$$

If $T$ is a bounded linear operator on $X$, then $M(T)$ denotes the operator on $X$ defined by

$$
M(T) x=J_{q}^{*} T^{*} J_{p} T x \quad \forall x \in X,
$$

where $T^{*}: X^{*} \rightarrow X^{*}$ is the adjoint operator of $T$, and $J_{p}, J_{q}^{*}$, respectively, are the duality mapping from $X$ to $X^{*}$ and $X^{*}$ to $X$, with $q=(p-1)^{-1} p$ and $p>1$ arbitrarily.

Received by the editors September 15, 1989 and, in revised form, April 23, 1990.

1980 Mathematics Subject Classification (1985 Revision). Primary 47A35, 47B38, 28 D99. 
Let $T_{n}: X \rightarrow X, n \geq 1$, be an arbitrary sequence of linear contractions (namely, $\left\|T_{n}\right\| \leq 1, n \geq 1$ ). In a recent paper [1], Akcoglu and Sucheston proved that $M\left(T_{n} \cdots T_{1}\right) f$ strongly converges in $L_{p}$ spaces $(1<p<\infty)$ as $n \rightarrow \infty$ for every element $f \in L_{p}$. This result has several interesting consequences and applications $[1,2,7]$ (in particular, it generalizes the well-known theorems of Stein [8], Rota [9], and Von Neumann [2]). In this note, we extend this result to the general cases of uniformly convex and uniformly smooth Banach spaces. Our argument will differ from that which Akcoglu and Sucheston used, allowing us to give an explicit estimate on the rate of convergence of $M\left(T_{n} \cdots T_{1}\right) x$.

\section{Preliminaries}

Recall that a Banach space $X$ is said to be uniformly convex if the modulus of $X$ that is defined by

$$
\delta_{X}(\varepsilon):=\inf \{1-\|x+y\| / 2:\|x\|=\|y\|=1,\|x-y\| \leq \varepsilon\}
$$

is positive for any $\varepsilon \in(0,2] ; X$ is said to is uniformly smooth (or equivalently, $X^{*}$ is uniformly convex) if the modulus of smoothness of $X$ defined by

$$
\rho_{X}(\tau):=\sup \left\{\frac{\|x+y\|}{2}+\frac{\|x-y\|}{2}-1:\|x\|=1,\|y\| \leq \tau\right\}
$$

satisfies the equality $\lim _{\tau \rightarrow \infty} \rho_{X}(\tau) / \tau=0$. It is known [3, Proposition 1.e.2] that there exists a complete duality between uniform convexity and uniform smoothness: $X$ is uniformly convex (smooth) if and only if $X^{*}$ is uniformly smooth (convex). We shall employ the following characteristics of these spaces that were recently established by the authors in [6]:

Lemma 1 [6]. Let $X$ be a uniformly convex Banach space and $k>1$ be an arbitrary real number. Then there exists a positive constant $c_{1}$ such that

$$
\|x+y\|^{k} \geq\|x\|^{k}+k\left\langle j_{k} x, y\right\rangle+\sigma_{k}(x, y) \quad \forall j_{k} x \in J_{k} x, x, y \in X,
$$

where

$$
\sigma_{k}(x, y)=c_{1} \int_{0}^{1} \frac{(\|x+t y\| \vee\|x\|)^{k}}{t} \delta_{X}\left(\frac{t\|y\|}{2(\|x+t y\| \vee\|x\|)}\right) d t .
$$

Lemma 2 [6]. Let $X$ be a uniformly smooth Banach space. Then for any $k>1$, the duality mapping $J_{k}$ is single-valued, and there exists a positive constant $c_{2}$ such that

$$
\left\|J_{k} x-J_{k} y\right\| \leq c_{2}(\|x\| \vee\|y\|)^{k-1} \bar{\rho}_{X}\left(\frac{\|x-y\|}{\|x\| \vee\|y\|}\right) \quad \forall x, y \in X
$$

where $\bar{\rho}_{X}(0)=0$ and

$$
\bar{\rho}_{X}(\tau)=\rho_{X}(\tau) / \tau \quad \forall \tau>0
$$


We also need the following consequence of Lemmas 1 and 2:

Lemma 3. Let $X$ be a uniformly smooth Banach space and $S: X \rightarrow X$ be a linear contraction. Then for any $k, p \in(1, \infty)$,

$$
\left\|S^{*} J_{p} S x-J_{p} x\right\| \leq\|x\|^{p-1} \varphi_{X^{*}}^{-1}\left(\frac{k}{c_{1}}\|x\|^{-p}\left(\|x\|^{p}-\|S x\|^{p}\right)\right) \quad \forall 0 \neq x \in X
$$

where

$$
\varphi_{X^{*}}(t)=\int_{0}^{t / 2} \delta_{x^{*}}(\xi) / \xi d \xi
$$

and $c_{1}$ is the constant determined by $X^{*}$ in Lemma 1.

Proof. Since $J_{p}$ is single-valued, $X^{*}$ is uniformly convex, and

$$
\begin{aligned}
\left\|J_{p} x+t\left(S^{*} J_{p} S x-J_{p} x\right)\right\| & =\left\|(1-t) J_{p} x+t S^{*} J_{p} S x\right\| \\
& \leq \operatorname{Max}\left\{\left\|J_{p} x\right\|,\left\|S^{*} J_{p} S x\right\|\right\} \\
& \leq\|x\|^{p-1}=\left\|J_{p} x\right\| \quad \forall 0 \leq t \leq 1,
\end{aligned}
$$

we have

$$
\begin{aligned}
\sigma_{k}\left(J_{p} x, S^{*} J_{p} S x-J_{p} x\right) & =c_{1} \int_{0}^{1} \frac{\left\|J_{p} x\right\|^{k}}{t} \delta_{X^{*}}\left(\frac{t\left\|S^{*} J_{p} S x-J_{p} x\right\|}{2\left\|J_{p} x\right\|}\right) d t \\
& =c_{1}\left\|J_{p} x\right\|^{k} \int_{0}^{\frac{\left\|S^{*} J_{p} S x-J_{p} x\right\|}{2\left\|J_{p} x\right\|^{\|}}} \delta_{X^{*}}(\xi) / \xi d \xi \\
& =c_{1}\|x\|^{k(p-1)} \varphi_{X^{*}}\left(\frac{\left\|S^{*} J_{p} S x-J_{p} x\right\|}{\|x\|^{p-1}}\right)
\end{aligned}
$$

where $\varphi_{X^{*}}$, as defined in (3), is a strictly increasing positive function. Then by Lemma 1 , we get

$$
\begin{aligned}
\left\|S^{*} J_{p} S x\right\|^{k}= & \left\|J_{p} x+S^{*} J_{p} S x-J_{p} x\right\|^{k} \\
\geq & \left\|J_{p} x\right\|^{k}+k\left\langle j_{k}^{*}\left(J_{p} x\right), S^{*} J_{p} S x-J_{p} x\right\rangle \\
& +c_{1}\|x\|^{k(p-1)} \varphi_{X^{*}}\left(\frac{\left\|S^{*} J_{p} S x-J_{p} x\right\|}{\|x\|^{p-1}}\right) .
\end{aligned}
$$

It is known from $\left[4 ; 6\right.$, Lemma 4] that $J_{k}^{*} x^{*}=\left\|x^{*}\right\|^{k-q} J_{q}^{*} x^{*}$ and $J_{q}^{*} x^{*}=J_{p}^{-1} x^{*}$ for any $x^{*} \in X^{*}, k, p \in(1, \infty)$, and $q=(p-1)^{-1} p$. Therefore,

$$
\begin{aligned}
\left\|S^{*} J_{p} S x\right\|^{k} \geq & \left\|J_{p} x\right\|^{k}+k\left\|J_{p} x\right\|^{k-q}\left\langle x, S^{*} J_{p} S x-J_{p} x\right\rangle \\
& +c_{1}\|x\|^{k(p-1)} \varphi_{X^{*}}\left(\frac{\left\|S^{*} J_{p} S x-J_{p} x\right\|}{\|x\|^{p-1}}\right) \\
= & \|x\|^{k(p-1)}+k\|x\|^{(p-1)(k-q)}\left(\|S x\|^{p}-\|x\|^{p}\right) \\
& +c_{1}\|x\|^{k(p-1)} \varphi_{X^{*}}\left(\frac{\left\|S^{*} J_{p} S x-J_{p} x\right\|}{\|x\|^{p-1}}\right) .
\end{aligned}
$$


Since

$$
\left\|S^{*} J_{p} S x\right\|^{k} \leq\left\|J_{p} S x\right\|^{k}=\|S x\|^{k(p-1)} \leq\|x\|^{k(p-1)},
$$

it follows that

$$
c_{1}\|x\|^{k(p-1)} \varphi_{X^{*}}\left(\frac{\left\|S^{*} J_{p} S x-J_{p} x\right\|}{\|x\|^{p-1}}\right) \leq k\|x\|^{k(p-1)-p}\left(\|x\|^{p}-\|S x\|^{p}\right)
$$

that is,

$$
\left\|S^{*} J_{p} S x-J_{p} x\right\| \leq\|x\|^{p-1} \varphi_{X^{*}}^{-1}\left(\frac{k}{c_{1}}\|x\|^{-p}\left(\|x\|^{p}-\|S x\|^{p}\right)\right),
$$

which completes the proof of the lemma. Q.E.D.

\section{RESULTS}

Theorem 4. Let $X$ be a uniformly convex and uniformly smooth Banach space, and $T_{n}: X \rightarrow X, n \geq 1$, be an arbitrary sequence of (linear) contractions. Then $M\left(T_{n} \cdots T_{1}\right) x$ converges strongly in $X$ as $n \rightarrow \infty$ for any $x \in X$. Furthermore, let $M(x)=\lim _{n \rightarrow \infty} M\left(T_{n} \cdots T_{1}\right) x$ and $m(x)=\lim _{n \rightarrow \infty}\left\|T_{n} \cdots T_{1} x\right\|$. Then the following estimate of the rate of convergence holds:

$$
\begin{gathered}
M(x)=0, \quad x=0, \\
\left\|M\left(T_{n} \cdots T_{1}\right) x-M(x)\right\| \leq \sigma(x)\|x\| \psi\left(1-\left(\frac{m(x)}{\left\|T_{n} \cdots T_{1} x\right\|}\right)^{p}\right), \quad x \neq 0,
\end{gathered}
$$

where

$$
\begin{aligned}
& \sigma(x)= \begin{cases}1, & 1<p \leq 2, \\
{[\|x\| / m(x)]^{p-q},} & p \geq 2,\end{cases} \\
& \psi(t)=c c_{2} \bar{\rho}_{X^{*}}\left(\varphi_{X^{*}}^{-1}\left(p t / c_{1}\right)\right) \quad \forall t>0,
\end{aligned}
$$

and $c$ is a constant determined by $X^{*}$.

Proof. For any $k>1$, it is easily seen that $J_{k}(\alpha x)=\operatorname{sign}(\alpha)|\alpha|^{k-1} J_{k} x$ and hence, $M(S)(\alpha x)=\alpha M(S) x$ for any linear operator $S$ on $X$ and for any scalar $\alpha$. Therefore, we will only consider $\|x\| \leq 1$ without loss of generality. Let $R_{n}=T_{n} \cdots T_{1}, S_{m}=T_{m} \cdots T_{n+1}$, and $g_{n}=R_{n} x$ for any $m>n \geq 1$. Then $R_{m}=S_{m} R_{n}, M\left(T_{n} \cdots T_{1}\right) x=J_{q}^{*} R_{n}^{*} J_{p} R_{n} x=J_{q}^{*} R_{n}^{*} J_{p} g_{n}$, and $M\left(T_{m} \cdots T_{1}\right) x=$ $J_{q}^{*} R_{m}^{*} J_{p} R_{m} x=J_{q}^{*} R_{n}^{*} S_{m}^{*} J_{p} S_{m} g_{n}=J_{q}^{*} R_{n}^{*} S_{m}^{*} J_{p} g_{m}$. Since the uniform convexity of $X$ implies uniform smoothness of $X^{*}$, by using Lemma 2 for $x:=R_{n}^{*} J_{p} g_{n}$, $y:=R_{n}^{*} S_{m}^{*} J_{p} g_{m}$, and $k:=q$, we obtain

$$
\begin{aligned}
\left\|M\left(T_{n} \cdots T_{1}\right) x-M\left(T_{m} \cdots T_{1}\right) x\right\|= & \left\|J_{q}^{*} R_{n}^{*} J_{p} g_{n}-J_{q}^{*} R_{n}^{*} S_{m}^{*} J_{p} g_{m}\right\| \\
\leq & c_{2}\left(\left\|R_{n}^{*} J_{p} g_{n}\right\| \vee\left\|R_{n}^{*} S_{m}^{*} J_{p} g_{m}\right\|\right)^{q-1} \\
& \cdot \bar{\rho}_{X^{*}}\left(\frac{\left\|R_{n}^{*} J_{p} g_{n}-R_{n}^{*} S_{m}^{*} J_{p} g_{m}\right\|}{\left\|R_{n}^{*} J_{p} g_{n}\right\| \vee\left\|R_{n}^{*} S_{m}^{*} J_{p} g_{m}\right\|}\right) .
\end{aligned}
$$


Since $\rho_{X^{*}}(\tau)$ is known to be positive, convex, and satisfy $\rho_{X^{*}}(0)=0$ [4] and $\rho_{X^{*}}(\tau) / \tau^{2}$ to be equivalent to a decreasing function [3, Proposition 1.e.5], $\bar{\rho}_{X^{*}}(\tau)$ is nondecreasing and there exists an absolute constant $c<\infty$ such that

$$
\rho_{X^{*}}(\tau) / \tau^{2} \leq c \rho_{X^{*}}(\eta) / \eta^{2} \quad \forall \tau>\eta>0 .
$$

Hence, it follows from the contraction property of $R_{n}^{*},(5),(6)$, and (2) that

$$
\begin{aligned}
\| M\left(T_{n}\right. & \left.\cdots T_{1}\right) x-M\left(T_{m} \cdots T_{1}\right) x \| \\
\leq & c_{2}\left(\left\|R_{n}^{*} J_{p} g_{n}\right\| \vee\left\|R_{n}^{*} S_{m}^{*} J_{p} g_{m}\right\|\right)^{q-1} \bar{\rho}_{X^{*}}\left(\frac{\left\|J_{p} g_{n}-S_{m}^{*} J_{p} g_{m}\right\|}{\left\|R_{n}^{*} J_{p} g_{n}\right\| \vee\left\|R_{n}^{*} S_{m}^{*} J_{p} g_{m}\right\|}\right) \\
= & c_{2} \frac{\left(\left\|R_{n}^{*} J_{p} g_{n}\right\| \vee\left\|R_{n}^{*} S_{m}^{*} J_{p} g_{m}\right\|\right)^{q-2}}{\left\|J_{p} g_{n}-S_{m}^{*} J_{p} S_{m} g_{n}\right\|^{-1}} \\
& \cdot\left[\frac{\left\|J_{p} g_{n}\right\|}{\left\|R_{n}^{*} J_{p} g_{n}\right\| \vee\left\|R_{n}^{*} S_{m}^{*} J_{p} g_{m}\right\|} \frac{\left\|J_{p} g_{n}-S_{m}^{*} J_{p} g_{m}\right\|}{\left\|J_{p} g_{n}\right\|}\right]^{-2} \\
& \cdot \rho_{X^{*}}\left(\frac{\left\|J_{p} g_{n}\right\|}{\left\|R_{n}^{*} J_{p} g_{n}\right\| \vee\left\|R_{n}^{*} S_{m}^{*} J_{p} g_{m}\right\|} \frac{\left\|J_{p} g_{n}-S_{m}^{*} J_{p} g_{m}\right\|}{\left\|J_{p} g_{n}\right\|}\right) \\
\leq & c c_{2}\left(\left\|R_{n}^{*} J_{p} g_{n}\right\| \vee\left\|R_{n}^{*} S_{m}^{*} J_{p} g_{m}\right\|\right)^{q-2}\left\|J_{p} g_{n}-S_{m}^{*} J_{p} S_{m} g_{n}\right\| \\
& \cdot\left[\frac{\left\|J_{p} g_{n}-S_{m}^{*} J_{p} g_{m}\right\|}{\left\|J_{p} g_{n}\right\|}\right]^{-2} \rho_{X^{*}}\left(\frac{\left\|J_{p} g_{n}-S_{m}^{*} J_{p} g_{m}\right\|}{\left\|J_{p} g_{n}\right\|}\right) \\
= & c c_{2}\left(\left\|R_{n}^{*} J_{p} g_{n}\right\| \vee\left\|R_{n}^{*} S_{m}^{*} J_{p} g_{m}\right\|\right)^{q-2}\left\|J_{p} g_{n}\right\| \bar{\rho}_{X^{*}}\left(\frac{\left\|J_{p} g_{n}-S_{m}^{*} J_{p} S_{m} g_{n}\right\|}{\left\|J_{p} g_{n}\right\|}\right) .
\end{aligned}
$$

(Here, we have assumed $\left\|J_{p} g_{n}\right\| \neq 0$ for any $n \geq 1$. If there exists some $n$ such that $\left\|J_{p} g_{n}\right\|=0$, then it is easy to see that $M\left(T_{n} \cdots T_{1}\right) x \rightarrow 0$. Consequently the conclusion has been proved already.) By using Lemma 3 for $x:=g_{n}$, $S:=S_{m}$, and $k:=p$, we then have

$$
\begin{aligned}
&\left\|M\left(T_{n} \cdots T_{1}\right) x-M\left(T_{m} \cdots T_{1}\right) x\right\| \\
& \leq c c_{2}\left(\left\|R_{n}^{*} J_{p} g_{n}\right\| \vee\left\|R_{n}^{*} S_{m}^{*} J_{p} g_{m}\right\|\right)^{q-2}\left\|J_{p} g_{n}\right\| \\
& \cdot \bar{\rho}_{X^{*}}\left(\varphi_{X^{*}}^{-1}\left(\frac{p}{c_{1}}\left\|g_{n}\right\|^{-p}\left(\left\|g_{n}\right\|^{p}-\left\|S_{m} g_{n}\right\|^{p}\right)\right)\right) \\
&=\left(\left\|R_{n}^{*} J_{p} g_{n}\right\| \vee\left\|R_{n}^{*} S_{m}^{*} J_{p} g_{m}\right\|\right)^{q-2}\left\|J_{p} g_{n}\right\| \psi\left(1-\left(\left\|g_{m}\right\| /\left\|g_{n}\right\|\right)^{p}\right),
\end{aligned}
$$

where $\psi(t)$, as defined in (4.b), is a strictly increasing positive function. Now we observe that $\lim _{n \rightarrow \infty}\left\|g_{n}\right\|=\lim _{n \rightarrow \infty}\left\|T_{n} \cdots T_{1} x\right\| \equiv m(x)$ exists, for $\left\{\left\|T_{n} \cdots T_{1} x\right\|\right\}$ clearly is a nonincreasing sequence of nonnegative numbers and, if $m(x)=0$, then, obviously, $\left\|M\left(T_{n} \cdots T_{1}\right) x\right\|=\left\|J_{q}^{*} R_{n}^{*} J_{p} R_{n} x\right\| \leq\left\|T_{n} \cdots T_{1} x\right\|$ $\rightarrow 0$ as $n \rightarrow \infty$; if $m(x) \neq 0$, then $\left\|g_{n}\right\|=\left\|T_{n} \cdots T_{1} x\right\| \geq m(x)>0$ for any 
$n \geq 1$, which in turn implies that

$$
\begin{aligned}
\left\|R_{n}^{*} J_{p} g_{n}\right\| & \geq\|x\|\left\|R_{n}^{*} J_{p} R_{n} x\right\| \geq\left\langle R_{n}^{*} J_{p} R_{n} x, x\right\rangle=\left\langle J_{p} R_{n} x, R_{n} x\right\rangle \\
& =\left\|J_{p} R_{n} x\right\|\left\|R_{n} x\right\|=\left\|g_{n}\right\|^{p} \geq[m(x)]^{p}>0 \quad \forall n \geq 1 ;
\end{aligned}
$$

hence, (7) implies in this case that

$$
\left\|M\left(T_{n} \cdots T_{1}\right) x-M\left(T_{m} \cdots T_{1}\right) x\right\| \rightarrow 0 \quad(\text { as } m>n \rightarrow \infty) .
$$

Thus, in either case, $\left\{M\left(T_{n} \cdots T_{1}\right) x\right\}$ is a Cauchy sequence for any $x \in X$. Consequently, $\lim _{m \rightarrow \infty} M\left(T_{m} \cdots T_{1}\right) x \equiv M(x)$ exists for every $x \in X$. Taking the limit as $m \rightarrow \infty$ in the two sides of the inequality (7) and noting that $\left\|M\left(T_{m} \cdots T_{1}\right) x\right\|=\left\|R_{n}^{*} S_{m}^{*} J_{p} g_{m}\right\|^{q-1}$, we then obtain

$$
\begin{aligned}
& \left\|M\left(T_{n} \cdots T_{1}\right) x-M(x)\right\| \\
& \quad \leq\left(\left\|R_{n}^{*} J_{p} g_{n}\right\| \vee[M(x)]^{p-1}\right)^{q-2}\left\|J_{p} g_{n}\right\| \psi\left(1-\left(m(x) /\left\|g_{n}\right\|\right)^{p}\right)
\end{aligned}
$$

Obviously, to complete the proof, it remains to show that

$$
\Delta \equiv\left(\left\|R_{n}^{*} J_{p} g_{n}\right\| \vee[M(x)]^{p-1}\right)^{q-2}\left\|J_{p} g_{n}\right\| \leq \sigma(x)\|x\| .
$$

Distinguish between two possible cases:

Case I. If $q \geq 2$, then from the fact that $\left\|R_{n}^{*} J_{p} g_{n}\right\| \leq\left\|J_{p} g_{n}\right\|=\left\|g_{n}\right\|^{p-1}=$ $\left\|T_{n} \cdots T_{1} x\right\|^{p-1} \leq\|x\|^{p-1}$, and that $\left\|M\left(T_{m} \cdots T_{1}\right) x\right\|=\left\|J_{q}^{*} R_{m}^{*} J_{p} g_{m}\right\|=$ $\left\|R_{m}^{*} J_{p} g_{m}\right\|^{q-1} \leq\|x\|^{(p-1)(q-1)}=\|x\|$, we get

$$
\begin{aligned}
\Delta & =\lim _{m \rightarrow \infty}\left[\left\|R_{n}^{*} J_{p} g_{n}\right\| \vee\left\|M\left(T_{n} \cdots T_{1}\right) x\right\|^{p-1}\right]^{q-2} \cdot\left\|J_{p} g_{n}\right\| \\
& \leq\|x\|^{(p-1)(q-2)}\left\|J_{p} g_{n}\right\| \leq\|x\|^{(p-1)(q-2)} \cdot\|x\|^{p-1}=\|x\|,
\end{aligned}
$$

which obviously establishes (9) in this case.

Case II. If $q<2$, then by means of the fact that (8) implies $\left\|R_{n}^{*} J_{p} g_{n}\right\| \geq$ $[m(x)]^{p} /\|x\|$ and $[M(x)]^{p-1}=\lim _{m \rightarrow \infty}\left\|R_{m}^{*} J_{p} g_{m}\right\| \geq[m(x)]^{p} /\|x\|$, we obtain

$$
\begin{aligned}
\Delta & \leq\left([m(x)]^{p} /\|x\|\right)^{q-2}\left\|J_{p} g_{n}\right\| \\
& \leq\left([m(x)]^{p} /\|x\|\right)^{q-2}\|x\|^{p-1}=[\|x\| / m(x)]^{p-q}\|x\| .
\end{aligned}
$$

Hence, (9) is justified in this case also. With this, the proof is complete. Q.E.D.

As a special case of Theorem 4, we have

Theorem 5. Let $X=l^{p}, L^{p}$, or $W_{m}^{p}, 1<p<\infty$, be the usual sequence spaces, function spaces, or Sobolev spaces, and $T_{n}: X \Rightarrow X, n \geq 1$, be an arbitrary sequence of (linear) contractions and $x \in X$. Then $M\left(T_{n} \cdots T_{1}\right) x$ converges strongly in $X$ as $n \rightarrow \infty$, with the estimate of the rate of convergence:

$$
\begin{gathered}
M(x)=0, \quad x=0, \\
\left\|M\left(T_{n} \cdots T_{1}\right) x-M(x)\right\| \leq k\|x\|\left[1-m(x) /\left\|T_{n} \cdots T_{1} x\right\|\right]^{\left(k_{1}-1\right) / k_{2}}, \quad x \neq 0,
\end{gathered}
$$


where $k$ is an absolute constant and $k_{1}, k_{2}$ are given by

$$
k_{1}=\left\{\begin{array}{ll}
2, & 1<p \leq 2, \\
q, & p \geq 2,
\end{array} \quad k_{2}= \begin{cases}q, & 1<p \leq 2, \\
2, & p \geq 2 .\end{cases}\right.
$$

Proof. It is known [3-5] that in these spaces the moduli of convexity and smoothness satisfy

$$
\begin{aligned}
& \delta_{X}(\varepsilon) \geq\left\{\begin{array}{ll}
\varepsilon^{2}(p-1) / 8, & 1<p \leq 2 \\
(1 / p)(\varepsilon / 2)^{p}, & p \geq 2
\end{array} \quad\left(X:=l^{p}, L^{p}, \text { or } W_{m}^{p}\right),\right. \\
& \rho_{X}(\tau) \leq\left\{\begin{array}{ll}
\tau^{p} / p, & 1<p \leq 2 \\
\tau^{2}(p-1) / 2, & p \geq 2
\end{array} \quad\left(X:=l^{p}, L^{p}, \text { or } W_{m}^{p}\right),\right.
\end{aligned}
$$

which imply that these spaces are both uniformly convex and uniformly smooth and, in particular, there exist constants $c_{1}, c_{2}>0$ such that

$$
\delta_{X^{*}}(\varepsilon) \leq c_{2} \varepsilon^{k_{2}}, \quad \rho_{X^{*}}(\tau) \leq c_{1} \tau^{k_{1}},
$$

where $k_{1}, k_{2}$ are defined as (10). Now by making use of these estimates, it is easily seen that Lemma 2 implies

$$
\left\|J_{q}^{*} x^{*}-J_{q} y^{*}\right\| \leq k_{1} c_{2}\left(\left\|x^{*}\right\| \vee\left\|y^{*}\right\|\right)^{q-k_{1}}\left\|x^{*}-y^{*}\right\|^{k_{1}-1},
$$

and Lemma 3 implies

$$
\left\|S^{*} J_{p} S x-J_{p} x\right\|^{k_{2}} \leq \frac{2^{k_{2}} k_{2} k}{c_{1} c_{2}}\|x\|^{(p-1)\left(k_{2}-q\right)}\left[\|x\|^{p}-\|S x\|^{p}\right] .
$$

Therefore, with all notations as in Theorem 4, we now have

$$
\begin{aligned}
\| M( & \left.T_{n} \cdots T_{1}\right) x-M\left(T_{m} \cdots T_{1}\right) x \| \\
= & \left\|J_{q}^{*} R_{n}^{*} J_{p} g_{n}-J_{q}^{*} R_{n}^{*} S_{m}^{*} J_{p} g_{m}\right\| \\
\leq & c_{2} k_{1}\left(\left\|R_{n}^{*} J_{p} g_{n}\right\| \vee\left\|R_{n}^{*} S_{m}^{*} J_{p} g_{m}\right\|\right)^{q-k_{1}}\left\|R_{n}^{*} J_{p} g_{n}-R_{n}^{*} S_{m}^{*} J_{p} g_{m}\right\|^{k_{1}-1} \\
\leq & c_{2} k_{1}\left(\left\|R_{n}^{*} J_{p} g_{n}\right\| \vee\left\|R_{n}^{*} S_{m}^{*} J_{p} g_{m}\right\|\right)^{q-k_{1}}\left\|S_{m}^{*} J_{p} S_{m} g_{n}-J_{p} g_{n}\right\|^{k_{1}-1} \\
\leq & c_{2} k_{1}\left(\left\|R_{n}^{*} J_{p} g_{n}\right\| \vee\left\|R_{n}^{*} S_{m}^{*} J_{p} g_{m}\right\|\right)^{q-k_{1}} \\
& \cdot\left[\frac{2^{k_{2}} k_{2} k}{c_{1} c_{2}}\left\|g_{n}\right\|^{(p-1)\left(k_{2}-q\right)}\left(\left\|g_{n}\right\|^{p}-\left\|S_{m} g_{n}\right\|^{p}\right)\right]^{\left(k_{1}-1\right) / k_{2}} \\
= & c_{2} k_{1}\left(\left\|R_{n}^{*} J_{p} g_{n}\right\| \vee\left\|R_{n}^{*} S_{m}^{*} J_{p} g_{m}\right\|\right)^{q-k_{1}} \\
& \cdot\left[\frac{2^{k_{2}} k_{2} k_{1}}{c_{1} c_{2}}\left\|g_{n}\right\|^{(p-1) k_{2}}\left(1-\frac{\left\|S_{m} g_{n}\right\|^{p}}{\left\|g_{n}\right\|^{p}}\right)\right]^{\left(k_{1}-1\right) / k_{2}}
\end{aligned}
$$

Since $k_{1} \leq q \leq k_{2}$ from (10) and $\left\|R_{n}^{*} J_{p} g_{n}\right\| \leq\left\|g_{n}\right\|^{p-1} \leq\|x\|^{p-1}$, it follows that

$$
\left\|M\left(T_{n} \cdots T_{1}\right) x-M\left(T_{m} \cdots T_{1}\right) x\right\| \leq k\|x\|\left[1-\left\|S_{m} g_{n}\right\|^{p} /\left\|g_{n}\right\|^{p}\right]^{\left(k_{1}-1\right) / k_{2}}
$$

for any $m>n \geq 1, x \neq 0$, and some constant $k>0$. From this, the conclusion of Theorem 5 obviously follows. This completes the proof. Q.E.D. 


\section{REFERENCES}

1. M. A. Akcoglu and L. Sucheston, An alternating procedure for operators on $L_{p}$ spaces, Proc. Amer. Math. Soc. 99 (1987), 555-558.

2. J. Von Neumann, Functional operators, vol. 2, Princeton Univ. Press, Princeton, NJ, 1950.

3. J. Lindenstrauss and L. Tzafriri, Classical Banach spaces II, Springer-Verlag, Berlin, 1979.

4. J. Diestel, Geometry of Banach space-selected topics, Lecture Notes in Math., vol. 485, Springer-Verlag, Berlin, 1975.

5. Ya. I. Al'ber and A. I. Notik, Geometric properties of Banach spaces and approximate method for solving nonlinear operator equations, Soviet Math. Dokl. 29 (1984), 611-615.

6. Zong-ben Xu and G. F. Roach, Characteristic inequalities of uniformly convex and uniformly smooth Banach spaces, J. Math. Anal. Appl. (to appear).

7. F. Deutsch, Rate of convergence of the method of alternating projections, Parametric Optimization and Approximation (Oberwolfach, 1983), Internat. Schriftenreihe Numer. Math., vol. 72, Birkhauser, Basel, 1985, pp. 96-107.

8. E. Stein, On the maximal ergodic theorem, Proc. Nat. Acad. Sci. USA 47 (1961), 1894-1897.

9. G.-C. Rota, An "Alternierende Verfahren" for general positive operators, Bull. Amer. Math. Soc. 68 (1962), 95-102.

Department of Mathematics, XI'AN Jiaotong University, Xi'an, China

Department of Mathematics, University of Strathclyde, Glasgow, United Kingdom 